\title{
USP14 regulates autophagy by suppressing K63 ubiquitination of Beclin 1
}

\author{
Daichao Xu ${ }^{1}$ Bing Shan, ${ }^{1}$ Huawang Sun, ${ }^{1}$ Juan Xiao, ${ }^{1,3}$ Kezhou Zhu, ${ }^{1}$ Xingxing Xie, ${ }^{1}$ Xingyan Li, ${ }^{1}$ \\ Wei Liang, ${ }^{1}$ Xiaojuan Lu, ${ }^{1}$ Lihui Qian, ${ }^{1}$ and Junying Yuan ${ }^{1,2}$ \\ ${ }^{1}$ Interdisciplinary Research Center on Biology and Chemistry, Shanghai Institute of Organic Chemistry, Chinese Academy of \\ Sciences, Shanghai 201210, China, ${ }^{2}$ Department of Cell Biology, Harvard Medical School, Boston, Massachusetts 02115, USA
}

The ubiquitin-proteasome system (UPS) and autophagy are two major intracellular degradative mechanisms that mediate the turnover of complementary repertoires of intracellular proteomes. Simultaneously activating both UPS and autophagy might provide a powerful strategy for the clearance of misfolded proteins. However, it is not clear whether UPS and autophagy can be controlled by a common regulatory mechanism. K48 deubiquitination by USP14 is known to inhibit UPS. Here we show that USP14 regulates autophagy by negatively controlling K63 ubiquitination of Beclin 1. Furthermore, we show that activation of USP14 by Akt-mediated phosphorylation provides a mechanism for Akt to negatively regulate autophagy by promoting K63 deubiquitination. Our study suggests that Akt-regulated USP14 activity modulates both proteasomal degradation and autophagy through controlling K48 and K63 ubiquitination, respectively. Therefore, regulation of USP14 provides a mechanism for Akt to control both proteasomal and autophagic degradation. We propose that inhibition of USP14 may provide a strategy to promote both UPS and autophagy for developing novel therapeutics targeting neurodegenerative diseases.

[Keywords: USP14; autophagy; Beclin 1; Akt]

Supplemental material is available for this article.

Received June 6, 2016; revised version accepted July 25, 2016.

Autophagy and the ubiquitin-proteasome system (UPS) are two major intracellular degradative mechanisms that function in a complementary manner. UPS mediates the degradation of short-lived proteins conjugated with K48 ubiquitin chains (Komander and Rape 2012). On the other hand, autophagy mediates the turnover of long-lived proteins and intracellular organelles encapsulated in autophagosomes that eventually fuse with lysosomes to allow degradation by lysosomal proteases. Ubiquitination is also involved as a signaling mechanism in targeting both protein substrates and organelles such as depolarized mitochondria for degradation by autophagy (Sarraf et al. 2013; Ordureau et al. 2014). Ubiquitination of protein substrates is a reversible process, as ubiquitin chains can be removed by deubiquitinating enzymes (DUBs). Deubiquitination is an important negative regulatory mechanism for reducing the levels of protein ubiquitination. Ubiquitin-specific protease-14 (USP14), a DUB reversibly associated with the proteasome, has been shown to negatively regulate the activity of proteasomes by trimming K48 ubiquitin chains on proteasome-bound substrates (Borodovsky et al. 2001; Koulich et al. 2008; Lee et al. 2010). USP14 can also be activated by Akt-mediated phosphory-

${ }^{3}$ Present address: Youjiang Medical University for Nationalities, Guangxi 533000, China.

Corresponding author: jyuan@hms.harvard.edu

Article is online at http://www.genesdev.org/cgi/doi/10.1101/gad.285122. 116. lation, which promotes its deubiquitinating activity for both K48 and K63 ubiquitin linkages (Xu et al. 2015a). The activity of USP14 in deubiquitinating K63 ubiquitin linkages is likely to be physiologically relevant, as inhibition of USP14 in vivo leads to increases in the levels of K63-linked ubiquitin conjugates in both spinal cords and neurons (Vaden et al. 2015). However, the mechanism by which USP14 regulates K63 ubiquitination in control of cellular processes and its functional significance are not well characterized.

Both UPS and autophagy are implicated in the removal and degradation of misfolded proteins that play critical roles in the pathogenesis of neurodegenerative diseases (Ciechanover and Kwon 2015). Since UPS and autophagy mediate the turnover of complementary repertoires of intracellular proteomes, simultaneously activating both UPS and autophagy might provide a powerful strategy for the clearance of misfolded proteins. However, UPS and autophagy have not been demonstrated to share a common regulatory mechanism. Here, we explore the mechanism and function of the K63 deubiquitinating activity of USP14. We show that USP14 can regulate

(C) $2016 \mathrm{Xu}$ et al. This article is distributed exclusively by Cold Spring Harbor Laboratory Press for the first six months after the full-issue publication date (see http://genesdev.cshlp.org/site/misc/terms.xhtml). After six months, it is available under a Creative Commons License (Attribution-NonCommercial 4.0 International), as described at http:// creativecommons.org/licenses/by-nc/4.0/. 
autophagy by controlling K63 ubiquitination of Beclin 1. Furthermore, we show that activation of USP14 by Akt-mediated phosphorylation provides a mechanism for Akt to negatively regulate autophagy by removing K63 ubiquitin chains from Beclin 1. Our study suggests that USP14 regulates both proteasomal degradation and autophagy through controlling K48 and K63 ubiquitination, respectively. Our results suggest that USP14 regulates both proteasomal and autophagic degradation. Therefore, inhibition of USP14 might provide a mechanism to promote both UPS and autophagy.

\section{Results}

\section{USP14 negatively regulates autophagy}

USP14 was identified as a gene that, when knocked down, led to up-regulation of autophagy in a siRNA screen targeting 127 DUBs (Fig. 1A). To confirm this finding, we transiently transfected $\mathrm{H} 4$ cells with siRNAs targeting USP14 and found that reduction of USP14 expression led to increased autophagy as detected by up-regulation of LC3-II protein levels and down-regulation of SQSTM1/p62 protein levels (Fig. 1B). The reduction of p62 protein levels could be restored by treatment with E64D (Mizushima et al. 2010), a lysosomal inhibitor that blocks autophagic flux, which is indicated by further increases in the levels of LC3-II (Fig. 1C). In contrast, treatment with MG132, an inhibitor of proteasomal degradation, had no effect on p62 degradation when USP14 was knocked down (Supplemental Fig. S1A). Thus, these results suggest that inhibition of USP14 promotes autophagic flux.

To further verify the role of USP14 in autophagy, we established a stable USP14 knockdown H4 cell line. Consistent with induction of autophagy, the levels of LC3-II were higher and the levels of p62 were lower in this USP14 knockdown cell line compared with that of the
A

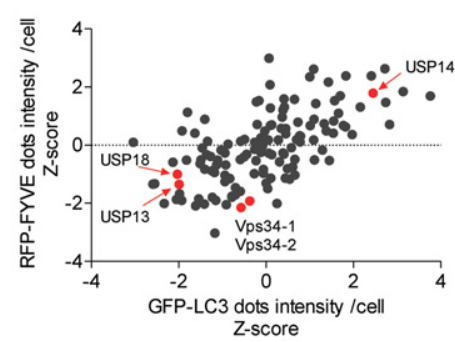

C

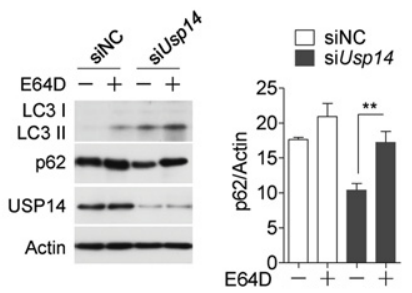

E
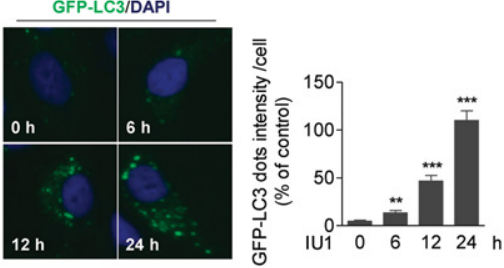

D
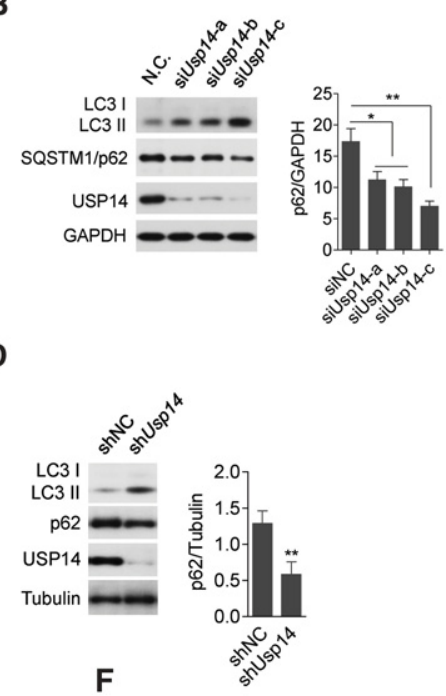

H

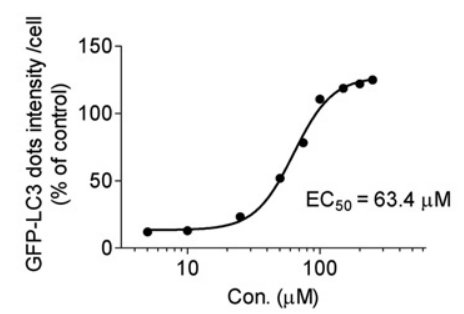

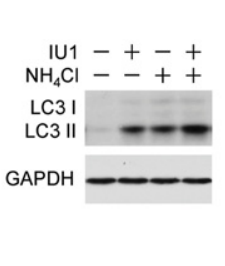

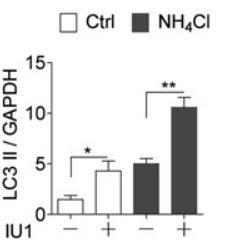

Figure 1. USP14 negatively regulates autophagy. $(A)$ Diagram of DUB siRNA screening data in H4-GFPLC3 cells and H4-RFP-FYVE cells in which two known regulators of autophagy, USP13 (Liu et al. 2011) and USP18 (Xu et al. 2015b), are indicated as positive controls. Vps34 was knocked down as a positive control in two replicates. $Z$-scores were calculated based on the plate median (controls excluded) and median absolute deviation, with $Z$-score $=($ cell score - median plate score)/(plate median absolute deviation $\times 1.4826$ ) (Lipinski et al. 2010). (B) Usp14 knockdown induces autophagy. $\mathrm{H} 4$ cells were transfected with control siRNA or three unique siRNAs targeting Usp14. Seventy-two hours after transfection, the cells were harvested for Western blot analysis. (NC) Nontarget siRNA control. Ratios of p62/GAPDH were calculated and are shown at the right. Results are shown as means \pm SD of three independent sets of experiments. $\left({ }^{*}\right) P<0.05$; $\left(^{* *}\right) P<0.01$. (C) USP14 regulates autophagic flux. $\mathrm{H} 4$ cells with Usp14 knockdown were treated with $10 \mu \mathrm{M}$ E64D for $6 \mathrm{~h}$ and harvested for Western blotting. Ratios of p62/Actin were calculated and are shown at the right. Results are shown as means \pm SD of three independent sets of experiments. $(D) \mathrm{H} 4$ cells with Usp14 stably knocked down were harvested for Western blotting. Ratios of p62/Tubulin were calculated and are shown at the right. Results are shown as means $\pm \mathrm{SD}$ of three independent sets of experiments. (E) Pharmacological inhibition of USP14 promotes autophagy. H4-GFPLC3 cells were treated with $50 \mu \mathrm{M} I U 1$ for the indicated periods of times. Images of the cells were collected using an ArrayScan HCS 4.0 reader. Representative cells are shown. The average spot intensity in 1000 cells from each indicated sample was determined. Data are displayed as means \pm SD of the spot intensity per cell. $\left.{ }^{* * *}\right) P<0.001$. $(F) \mathrm{H} 4$ cells were treated as in $E$ and harvested for Western blot analysis. $(G)$ The $\mathrm{EC}_{50}$ value for IU1 to induce autophagy was determined using the GFP-LC3 assay. H4-GFP-LC3 cells were treated with different concentrations of IU1 for $24 \mathrm{~h}$. The GFP-LC3 ${ }^{+}$puncta were quantified as in $E$. (H) IU1 induces autophagic flux. H4 cells were treated with $50 \mu \mathrm{M} \mathrm{IU} 1$ for $6 \mathrm{~h}$ in the presence or absence of $10 \mathrm{mM} \mathrm{NH}_{4} \mathrm{Cl}$ and harvested for Western blot analysis. Ratios of LC3-II/ GAPDH were calculated and are shown at the right. 
control under basal conditions (Fig. 1D). Furthermore, treatment with IU1 (Lee et al. 2010), an inhibitor of USP14, induced the accumulation of LC3-II in a timeand dose-dependent manner as determined by both GFPLC3 assay and Western blotting (Fig. 1E,F; Supplemental Fig. S1B). The $\mathrm{EC}_{50}$ of IU1 in inducing autophagy is 63.4 $\mu M$ as determined by GFP-LC3 assay (Fig. 1G), which is comparable with an $\mathrm{EC}_{50}$ of $62 \mu \mathrm{M}$ in inducing UPS activity (Lee et al. 2010) as determined by GFPu assay (Supplemental Fig. S1C; Bence et al. 2005; Xu et al. 2015a). Similarly, treatment with IU1 also induced autophagy in other cell lines tested, including Huh7, HeLa, HCT116, and mouse embryonic fibroblasts (MEFs) (Supplemental Fig. S1D-G). Consistent with its role as a negative regulator of autophagic flux, inhibition of USP14 by IU1 also induced the accumulation of LC3-II in the presence of $\mathrm{NH}_{4} \mathrm{Cl}$, a lysosomal inhibitor that blocks autophagic flux (Fig. 1H). Notably, the ability of IU1 to induce autophagy was blocked after USP14 knockout in H4 cells, suggesting that the activity of IU1 to induce autophagy requires USP14 (Supplemental Fig. S1H). Taken together, we conclude that USP14 is a negative regulator of autophagy, and, furthermore, inhibition of USP14 can simultaneously activate both autophagy and UPS.

\section{USP14 is important for Akt to inhibit autophagy}

Since USP14 is activated by Akt-mediated phosphorylation (Xu et al. 2015a), we next examined the possible role of USP14 as a downstream mediator of Akt in the control of autophagy. The expression of activated Akt (myristoylation signal [MGSSKSKPK]-attached Akt [Myr-Akt]) could reduce autophagy even in the presence of rapamycin, an inhibitor of mTOR, suggesting that Akt can inhibit autophagy in mTOR-independent mechanisms as report- ed (Supplemental Fig. S2; Wang et al. 2012). However, the ability of activated Akt to negatively regulate autophagy was significantly reduced when USP14 was knocked down (Fig. 2A), suggesting that USP14 is an important downstream mediator for Akt to regulate autophagy. Consistently, when the activity of Akt was inhibited by MK2206, the effect of USP14 knockdown in stimulating autophagy was reduced (Fig. 2B), further supporting the role of USP14 as a downstream mediator of Akt in suppressing autophagy. In addition, we tested the role of growth factors such as insulin-like growth factor 1 (IGF1) or epidermal growth factor (EGF), both of which are known to promote activation of Akt (Burgering and Coffer 1995). We found that treatment with IGF1 or EGF reduced the levels of autophagy in serum-starved wild-type H4-GFPLC3 cells, which was restored when the cells were treated with MK2206 (Fig. 2C). However, treatment with IGF1 or EGF failed to inhibit autophagy in H4-GFP-LC3 cells with USP14 stable knockdown (Fig. 2C). Taken together, these results suggest that USP14 plays a critical role as a downstream mediator for Akt to inhibit autophagy.

\section{Akt-mediated phosphorylation of USP14 is required for USP14 to inhibit autophagy}

We next tested the role of USP14 phosphorylation in Akt regulation of autophagy. To this end, expression vectors of wild-type USP14 or phosphorylation mutants of USP14 were stably transfected into Usp14 knockdown cells. As shown in Figure 3A, the expression of wild-type USP14 or a phosphorylation mimic mutant of USP14, USP14DD (S143D/S432D), but not the USP-AA (S143A/S432A) mutant, was able to reduce autophagy in USP14 stable knockdown cells. This result was further validated by using a GFP-LC3 assay in H4-GFP-LC3 cells with Usp14
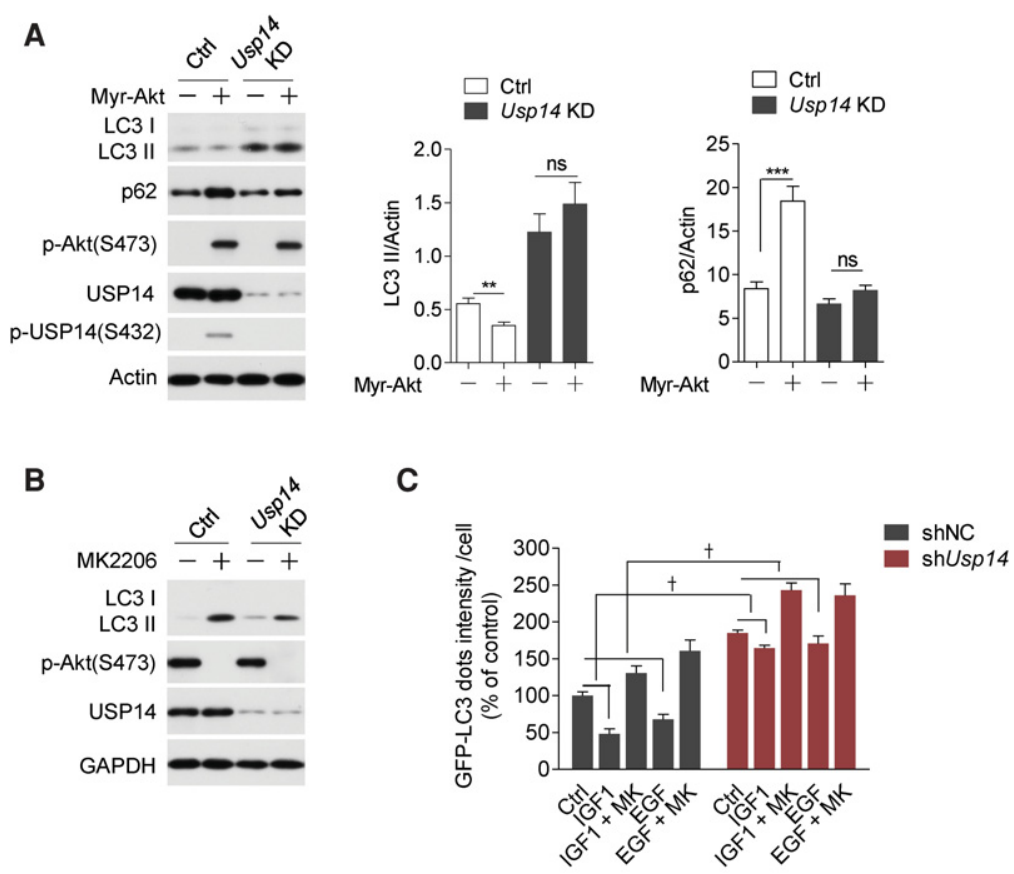

Figure 2. USP14 is important for Akt to inhibit autophagy. (A) Either wild-type or Usp14 stable knockdown (Usp14 KD) H4 cells were infected with a lentiviral vector expressing Myr-Akt for $24 \mathrm{~h}$. The cells were then harvested and subjected to Western blot analysis using the indicated antibodies. The ratios of LC3-II/Actin and p62/Acitn were calculated and are shown at the right. Results are shown as means \pm SD of three independent sets of experiments. (ns) Not significant. (B) Wild-type and Usp14 stable knockdown $\mathrm{H} 4$ cells were treated with $1 \mu \mathrm{M}$ MK2206 for $4 \mathrm{~h}$ and then harvested and analyzed by Western blotting using the indicated antibodies. $(C)$ Wild-type and Usp14 stable knockdown H4-GFPLC3 cells were serum-starved overnight and pretreated with $1 \mu \mathrm{M}$ MK2206 for $30 \mathrm{~min}$ before stimulation with $100 \mathrm{ng} / \mathrm{mL}$ IGF1 or $100 \mathrm{ng} / \mathrm{mL}$ EGF for 1 h. Images of the cells were collected using an ArrayScan HCS 4.0 reader. The average spot intensity in 1000 cells from each indicated sample was determined. Data are displayed as means \pm SD of the spot intensity per cell. ( + ) $P<0.01$ (ANOVA). 
A
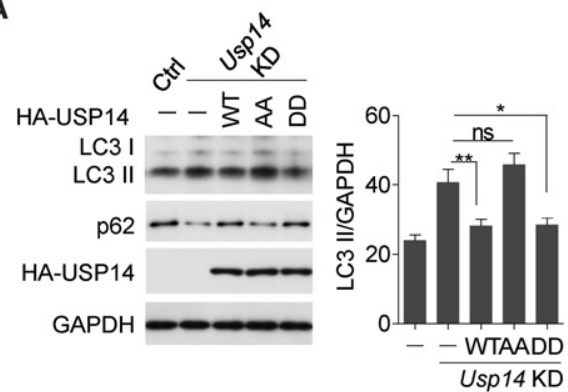

C
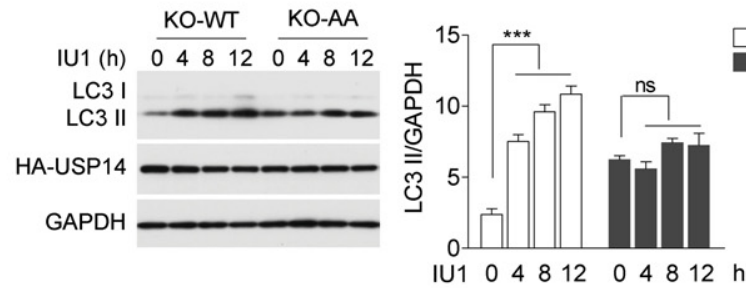

B

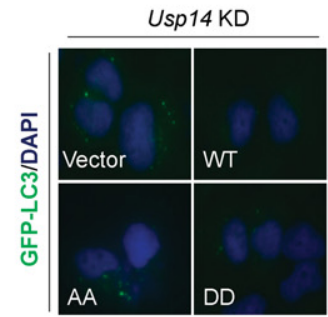

$\overline{\overline{0}}$

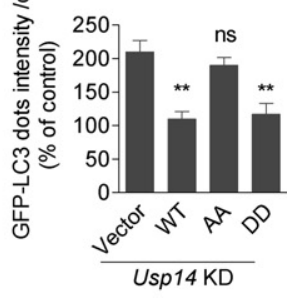

D

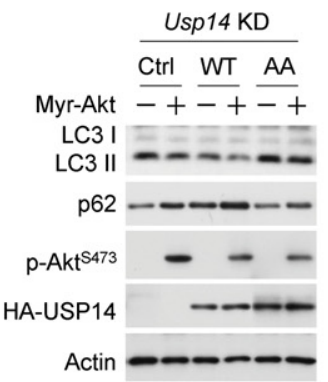

F
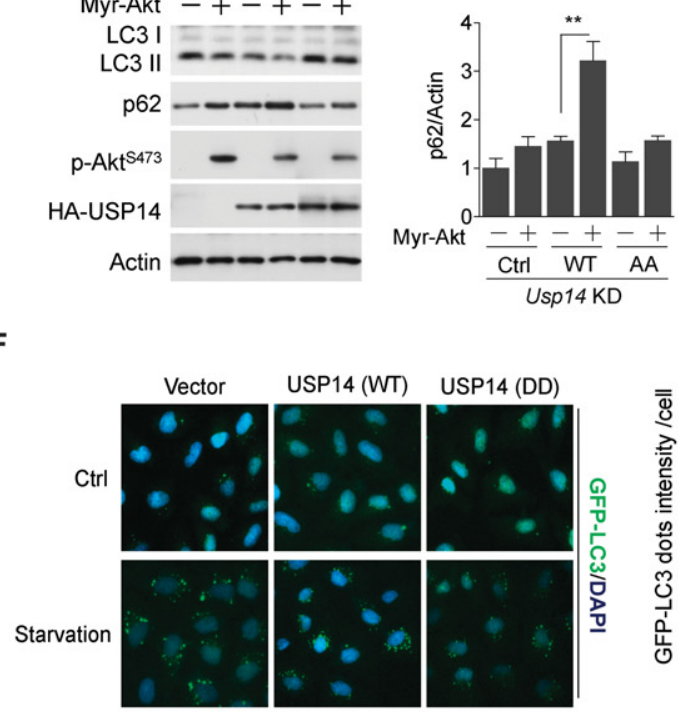

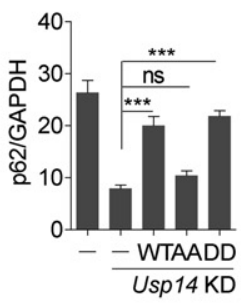

KO-WT KO-AA

\section{E}
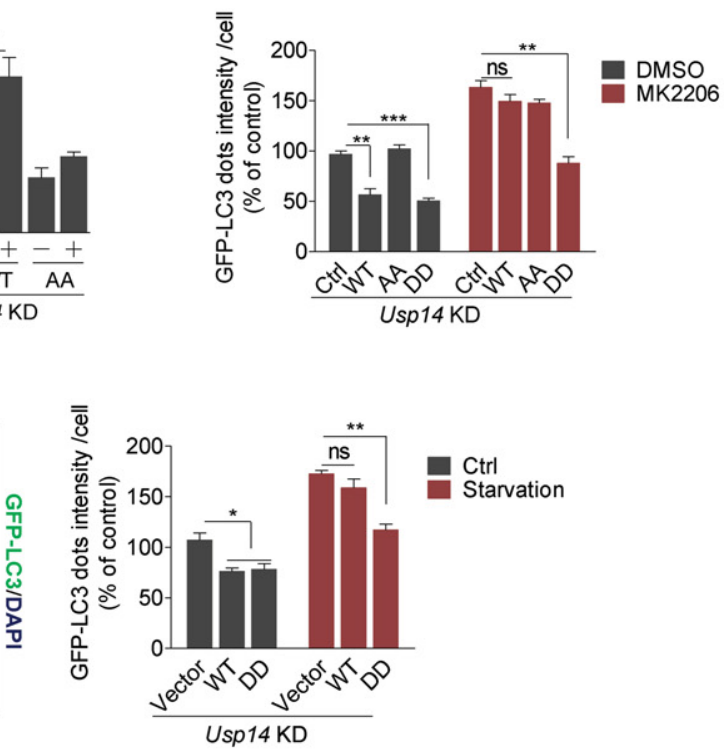

Figure 3. Akt-mediated phosphorylation of USP14 is required for USP14 to inhibit autophagy. (A) Usp14 stable knockdown H4 cells were infected with lentiviral vectors expressing wild-type USP14, the USP14-AA (S143A/S432A) mutant, or the USP14-DD (S143D/S432D) mutant as indicated for $24 \mathrm{~h}$. The cells were then harvested and subjected to Western blot analysis using the indicated antibodies. The ratios of LC3-II/GAPDH and p62/GAPDH were calculated and are shown at the right. Results are shown as means \pm SD of three independent sets of experiments. (B) Usp14 stable knockdown H4-GFP-LC3 cells were transfected as in $A$ and then imaged using an ArrayScan HCS 4.0 reader. The average spot intensity in 1000 cells from each indicated sample was determined. Bars represent mean \pm SEM of triplicate samples. (C) Usp14 ${ }^{-/-}$H4 cells expressing wild-type USP14 or the USP14-AA mutant were treated with $50 \mu M$ IU1 for indicated periods of times. Cells were then harvested for Western blot analysis. The ratios of p62/Actin were calculated and are shown at the right. (D) Usp14 stable knockdown H4 cells were infected with lentiviral vectors expressing either wild-type USP14 or the USP14-AA mutant in the presence or absence of Myr-Akt as indicated for $24 \mathrm{~h}$. The cells were then harvested and subjected to Western blot analysis using the indicated antibodies. The ratios of p62/Acitn were calculated and are shown at the right. Results are shown as means \pm SD of three independent sets of experiments. (ns) Not significant. (E) Usp14 stable knockdown H4-GFP-LC3 cells were infected with lentiviral vectors expressing wild-type USP14, the USP14-AA mutant, or the USP14-DD mutant as indicated for $20 \mathrm{~h}$ and then treated with or without $1 \mu$ M MK2206 for another $4 \mathrm{~h}$. The cells were imaged and quantified as in $B$. $(F)$ Usp14 stable knockdown H4-GFP-LC3 cells were infected with lentiviral-expressing vectors of wild-type USP14, the USP14-AA mutant, or the USP14-DD mutant as indicated for $12 \mathrm{~h}$ and then serum-starved for another $12 \mathrm{~h}$. The cells were imaged and quantified as in $B$. 
knockdown (Fig. 3B). Furthermore, inhibition of USP14 by IU1 in Usp14 $14^{-/-}$cells stably expressing wild-type USP14, but not the USP14-AA mutant, induced autophagy in a time-dependent manner (Fig. 3C), suggesting that phosphorylation of USP14, which promotes its activation (Xu et al. 2015a), is important for USP14 to inhibit autophagy.

To further validate the role of USP14 phosphorylation by Akt in the regulation of autophagy, we tested the effect of activated Akt in Usp14 knockdown cells expressing wild-type USP14 or the USP14-AA mutant. As shown in Figure 3D, the expression of activated Akt was able to inhibit autophagy in cells expressing wild-type USP14 but not in USP14-AA mutant cells. Consistently, when Akt was inhibited by MK2206 (Fig. 3E) or serum starvation (Fig. 3F; Franke et al. 1995), the expression of wild-type USP14 failed to inhibit autophagy, while that of the USP14-DD mutant could still inhibit autophagy, suggesting the importance of phosphorylation by Akt for USP14 to inhibit autophagy.
USP14 suppresses Vps34 activity by interacting with Beclin 1

We next characterized the mechanism by which USP14 regulated autophagy. PtdIn3P (phosphatidylinositol 3phosphate) is a key lipid signaling molecule involved in the nucleation of autophagosomes and is especially important for regulation of autophagy under normal nutritional conditions (Fig. 1A; Lipinski et al. 2010). The recruitment of FYVE-RFP to endosomal localized PtdIns3P has been used as an assay for measuring the cellular levels of PtdIns3P (Zhang et al. 2007). We found that knockdown of USP14 induced the formation of FYVE-RFP dots (Figs. 1A, 4A), suggesting that USP14 may negatively regulate Vps34 activity critical for the production of PtdIns3P under basal condition.

Since inhibition of USP14 promoted the degradation of a set of proteins through UPS (Xu et al. 2015a), we first considered the possibility that inhibition of USP14 promoted the degradation of an inhibitor of class III PI3 kinase
A

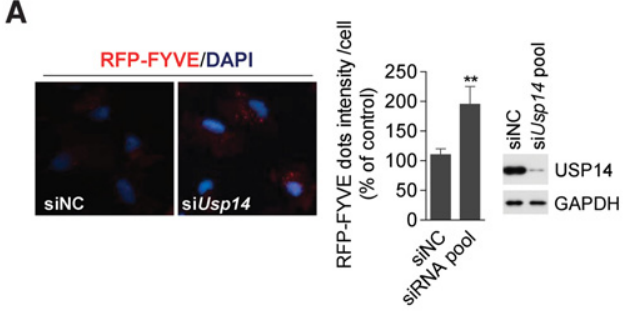

C

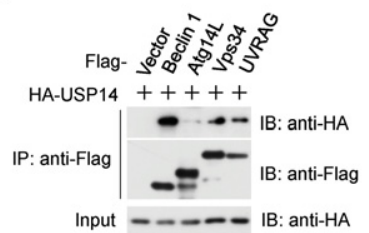

D

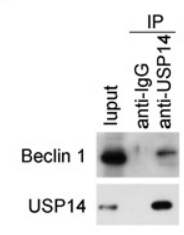

E

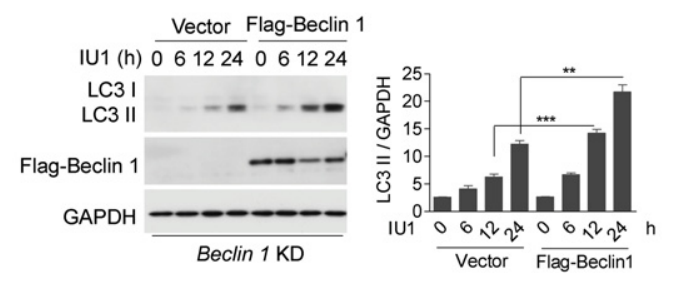

B

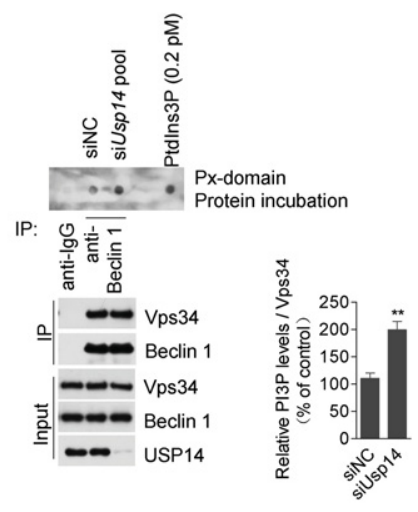

$\mathbf{F}$

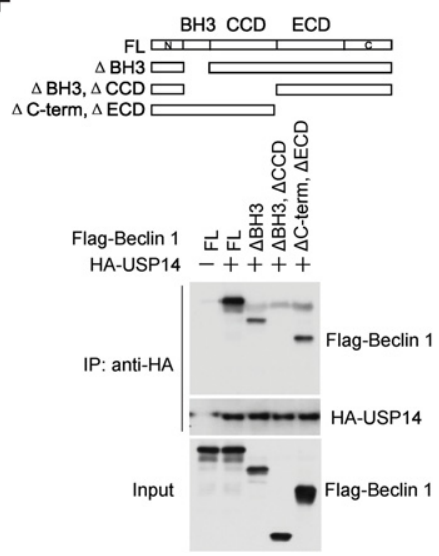

Figure 4. USP14 suppresses Vps34 activity by interacting with Beclin 1. (A) USP14 deficiency enhances Vps34 activity. H4RFP-FYVE cells were transfected with control siRNA or siRNA pools targeting Usp14. Seventy-two hours after transfection, the cells were imaged using an ArrayScan HCS 4.0 reader. Representative cells are shown. The average RFP-FYVE spot intensity in 1000 cells from each indicated sample was determined. The data are displayed as the means \pm SEM of the spot intensity per cell. USP14 knockdown efficiency is shown at the right. $(B)$ The Beclin 1-associated Vps34 lipid kinase assay. $\mathrm{H} 4$ cells $\left(2 \times 10^{7}\right.$ cells $)$ were lysed after being transfected with either a control siRNA or siRNA pools targeting Usp14 for $72 \mathrm{~h}$, and the cell lysates were incubated with anti-Beclin 1 antibody to precipitate the Beclin 1/Vps34 complex. The immunoprecipitates were separated into two parts: one for input detection and the other for kinase assays. The production of PtdIns3P (PI3P) was detected by protein-lipid blot assay. Lipids were extracted and applied onto a Hybond C-extra membrane. The commercial PtdIns3P was spotted as indicated for control. The levels of PtdIns3P were detected using the GST-PX-p40 domain protein, which binds to PtdIns3P and antiGST antibody. Ratios of PtdIns3P levels/ Vps34 were calculated and are shown.

PI3P (0.2 pM) was used as a positive control. (C) USP14 associates with Vps34 complexes. The expression vectors of HA-tagged USP14 and Flag-tagged Beclin 1, Vps34, Atg14L, or UVRAG were cotransfected into HEK293T cells, respectively, and immunoprecipitations (IP) were performed at $24 \mathrm{~h}$ after transfection. (D) $\mathrm{H} 4$ cells $\left(2 \times 10^{7}\right.$ cells) were lysed, the lysates were analyzed by immunoprecipitation with anti-IgG control or anti-USP14, and the immunocomplexes were analyzed by Western blot analysis. $(E) \mathrm{H} 4$ cells with Beclin 1 stable knockdown were virally infected with expression vectors for either control or Flag-tagged Beclin 1 for $12 \mathrm{~h}$ and then treated with $50 \mu \mathrm{M}$ IU1 for the indicated periods of times. The cells were harvested for Western blot analysis. The ratios of LC3-II/GAPDH were calculated and are shown at the right. $(F)$ Mapping the interacting regions of Beclin 1 with USP14. The expression vectors of Flag-tagged Beclin 1 truncations encoding the indicated regions were cotransfected with that of full-length USP14 into HEK293T cells and analyzed by immunoprecipitation and Western blotting. 
activity. However, no obvious candidate was identified in the list of proteins whose levels were reduced after USP14 knockout. We further experimentally tested whether inhibition of USP14 might promote the degradation of Rubicon, a well-established inhibitor of the Vps34 complex (Zhong et al. 2009). As shown in Supplemental Figure S3A, treatment with IU1 had no effect on the levels of Rubicon. To directly test the effect of USP14 knockdown and inhibition by IU1 on the activity of the Vps34/Beclin 1 complex in converting PtdIns to PtdIns3P, we conducted an in vitro kinase assay using the isolated Vps34/Beclin 1 complex and lipid blots (Fig. 4B; Supplemental Fig. S3B). These results showed that inhibition of USP14 by pharmacological means or siRNA-mediated knockdown led to activation of class III PI3 kinase activity; however, it was unlikely that this was accomplished by promoting the degradation of the inhibitory activity of Vps34 complexes.

To explore the mechanism by which USP14 regulates the production of PtdIns3P, we screened for the components of the Vps34 complex that can interact with USP14. We found that overexpressed USP14 showed a strong interaction with Beclin 1 (Fig. 4C; Supplemental Fig. S3C). Furthermore, we detected the interaction of endogenous Beclin 1 and USP14, both of which were predominantly present in the cytoplasm (Fig. 4D; Supplemental Fig. S3D). To test the possible role of Beclin 1 in USP14-regulated autophagy, a stable Beclin 1 knockdown $\mathrm{H} 4$ cell line was generated (Supplemental Fig. S3E; Xu et al. 2015b). As shown in Figure 4E, the effect of IU1 on the levels of LC3-II was smaller in this Beclin 1 knockdown cell line compared with that of the knockdown-reconstituted Beclin 1 cell line, suggesting that USP14 regulates autophagy in a Beclin 1-dependent manner.

To further characterize the interaction of USP14 with Beclin 1, we determined the domains of Beclin 1 that interact with USP14. Expression vectors of truncated Beclin 1 mutants were coexpressed with that of USP14 in HEK293T cells, and the interaction of USP14 with Beclin 1 mutants was analyzed by coimmunoprecipitation. The binding of USP14 with Beclin 1 was significantly reduced upon deletion of its CC domain (Fig. 4F), suggesting that the CC domain of Beclin 1 is important for the interaction with USP14.

\section{USP14 regulates Beclin 1 K63-linked ubiquitination}

Since knockdown or inhibition of USP14 had no effect on the levels of key components of Vps34 complexes (Supplemental Fig. S4), we hypothesized that USP14 might regulate autophagy through a nondegradative mechanism independently of K48 ubiquitination. Since USP14 can deubiquitinate K63 ubiquitin linkage (Jung et al. 2013; Xu et al. 2015a; Lee et al. 2016), we considered the possibility that USP14 may regulate autophagy by deubiquitinating K63 ubiquitinated proteins. To directly test this possibility, we performed a quantitative mass spectrometry analysis to compare the changes in K63 ubiquitinated proteins with H4 cells expressing USP14 wild type and those expressing vector only or the USP14-AA mutant (Fig. 5A). We established H4 cells sta- bly expressing Flag and His-double-tagged K63-only ubiquitin with vector only, USP14 wild type, or USP14-AA. K63 ubiquitinated proteins were first immunoprecipitated using anti-Flag from the cell lysis. The anti-Flag immunocomplexes were then denatured in $8 \mathrm{M}$ urea, and individual K63 ubiquitinated proteins were isolated by $\mathrm{Ni}^{2+}$ beads, which bind to the His tag. Using an isobaric tandem mass tag (TMT) labeling approach, our mass spectrometry analysis identified 19,503 peptides with high confidence $(q<0.01)$, corresponding to 2651 proteins with at least one unique peptide in each protein. Onethousand-six-hundred-six proteins were quantified in at least two of three replicates. Among those 1606 proteins, we found 1117 proteins that were significantly decreased $(P<0.05)$ from immunoprecipitation of K63 ubiquitinated proteins in $\mathrm{H} 4$ cells overexpressing wild-type USP14 compared with that in $\mathrm{H} 4$ cells expressing control vector. In this set of 1117 proteins, we found that the levels of 74 proteins were decreased at least 1.3 -fold, suggesting that these proteins may be substrates of K63 deubiquitination by USP14 (Fig. 5B, Lane 1).

In addition, we compared the mass spectrometry analysis of K63 ubiquitinated proteins from cells expressing wild-type USP14 with those expressing the USP14-AA mutant. Importantly, we identified a set of 59 proteins in common with the set of 74 ubiquitinated proteins $(80 \%)$ identified above from the comparison of wild-type USP14/vector. This set of 59 proteins was significantly increased $(P<0.05)$ from immunoprecipitation of K63 ubiquitinated proteins in cells overexpressing the USP14-AA mutant, an inactive form of USP14 (Xu et al. 2015a), compared with that of USP14 wild type (Fig. 5B, lanes 2,3). Interestingly, Beclin 1 was identified as a common top hit, with a twofold decrease on both lists of K63 ubiquitinated proteins that were altered in response to changes in the expression of wild type when compared with vector alone or the USP14-AA mutant. Since Beclin 1 is an essential component of Vps34 complexes, this result suggests that USP14 may regulate K63 ubiquitination of Beclin 1.

Next, we directly characterized the effect of USP14 on K63 ubiquitination of Beclin 1. We found that inhibition of USP14 by either siRNA knockdown or IU1 led to increases in the levels of K63 ubiquitination on endogenous Beclin 1 (Fig. 5C). Conversely, we found that overexpression of USP14 significantly reduced the K63, but not K48, ubiquitination levels of Beclin 1 (Fig. 5D). To demonstrate that USP14 can directly deubiquitinate Beclin 1 K63 ubiquitination, we performed in vitro deubiquitination assays. Incubation of purified activated USP14-S432Ebut not USP14 wild type, which is known to be inactive in vitro in the absence of proteasomes (Hu et al. 2005; Lee et al. 2010; Xu et al. 2015a)—with K63 ubiquitinated Beclin 1 in vitro led to its deubiquitination (Fig. 5E).

To characterize the mechanism underlying Beclin 1 K63 ubiquitination-mediated promotion of autophagy, we performed a mass spectrometry analysis to determine the interactome changes of Beclin 1 with or without K63 ubiquitination. Interestingly, we found that K63 ubiquitination of Beclin 1 increased its interaction with two well-characterized Beclin 1-binding partners- 
Xu et al.

A

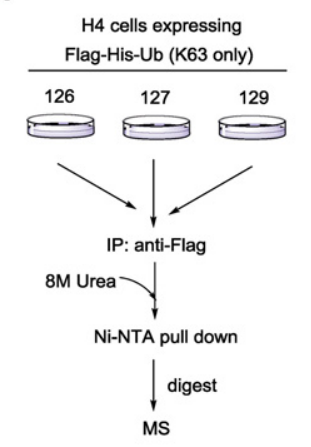

D

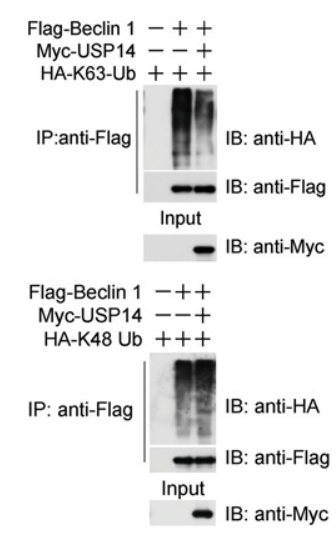

F

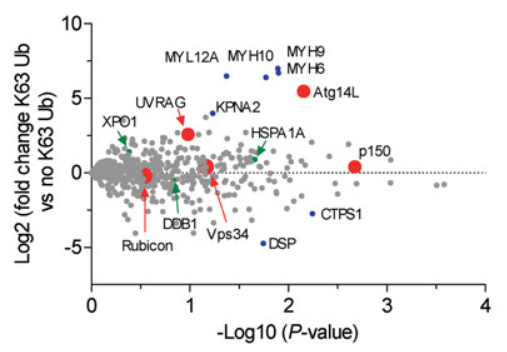

B
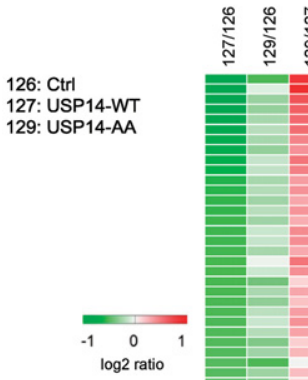

G
C

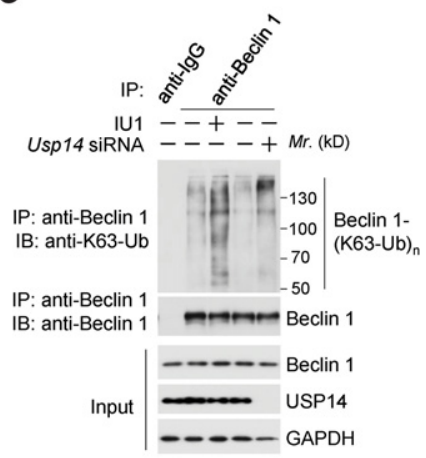

E

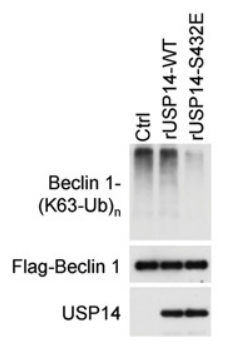

H

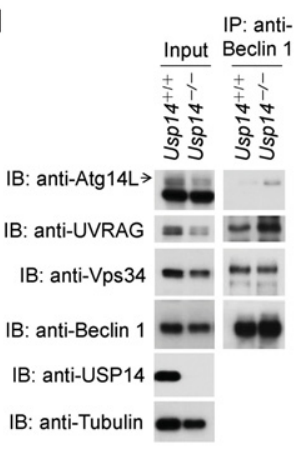

Figure 5. USP14 regulates Beclin 1 K63-linked ubiquitination. (A) Schematic representation of mass spectrometry assay to determine the changes in K63 ubiquitinated proteins in response to overexpression of USP14. (B) The quantitative analysis of ubiquitinated proteome changes in control cells or cells overexpressing wild-type USP14 or the USP14-AA mutant expressing Flag-His-K63-only ubiquitin were performed by TMT isobaric labeling followed by shotgun analysis. The heat map was plotted based on the set of 74 proteins that were significantly decreased $(P<0.05)$ at least 1.3 -fold from K63 ubiquitination immunoprecipitation in the presence of wild-type USP14 compared with control cells. The log base 2 of average ratios was plotted as indicated. (C) H4 cells $\left(2 \times 10^{7}\right.$ cells $)$ were transfected with siRNA pools targeting Usp14 for $72 \mathrm{~h}$ or treated with $50 \mu \mathrm{M}$ IU1 for $24 \mathrm{~h}$ and then lysed, and the lysates were incubated with antiBeclin 1 antibody followed by Western blot analysis to determine the K63 ubiquitination on Beclin 1. (D) USP14 reduced the K63, but not K48, ubiquitination levels of Beclin 1. HEK293T cells were transfected with expression vectors of HA-tagged K63-only ubiquitin or K48only ubiquitin and Flag-tagged Beclin 1 as indicated with or without Myc-tagged USP14 and cultured for $24 \mathrm{~h}$. The cell lysates were immunoprecipitated with anti-Flag antibody, and the immunocomplexes were analyzed by Western blotting with anti-HA antibody. $(E)$ In vitro K63-linked deubiquitination of Beclin 1 by USP14. K63 ubiquitinated Beclin 1 was incubated with purified recombinant USP14 or USP14-S432E in vitro and then blotted with anti-HA antibody. (F) A scatter plot depicting interactome changes of Beclin 1 identified and quantified in a quantitative proteomics experiment. Proteins were plotted as a function of fold change with K63 ubiquitin relative to the control. Some of the known Beclin 1 interactors (from BioGRID, http://thebiogrid.org) are highlighted in red and green. Some of the unknown binding targets with significant fold changes are highlighted in blue. The targets in Beclin 1-containing Vps34 complexes (Vps34, p150, Atg14L, UVRAG, and Rubicon) are highlighted by larger red dots. (G) K63 ubiquitination promotes the association of Atg14L and UVRAG with Beclin 1. Control H4 cells or K63 ubiquitin stably expressing H4 cells were harvested, the cell lysates were incubated with anti-Beclin 1 antibody to precipitate the Beclin 1/Vps34 complex, and the immunocomplexes were analyzed by Western blotting with the

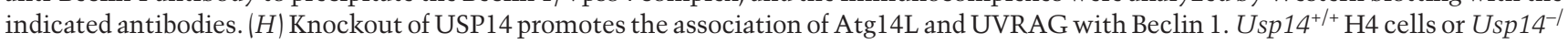
${ }^{-} \mathrm{H} 4$ cells were harvested, the cell lysates were incubated with anti-Beclin 1 antibody to precipitate the Beclin $1 / \mathrm{Vps} 34$ complex, and the immunocomplexes were analyzed by Western blotting with the indicated antibodies. 
Atg14L and UVRAG - that are important regulators of Vps34 complex activity (Fig. 5F; Itakura et al. 2008; Matsunaga et al. 2009; Zhong et al. 2009; Russell et al. 2013). On the other hand, the association of Vps34, p150, and Rubicon with Beclin 1 did not change significantly (Fig. 5F). These results suggest that K63 ubiquitination of Beclin 1 may promote autophagy through the increased association of Atg14L and UVRAG with Beclin 1.

To verify these results, we then performed immunoprecipitation-immunoblot assays to determine the interaction of Atg14L and UVRAG with Beclin 1 in the presence of K63 ubiquitin. Consistent with the results from mass spectrometry analysis, we found that K63 ubiquitination promoted the interaction of Beclin 1 with Atg14L and UVRAG but not with Vps34 (Fig. 5G). Furthermore, we found that the interaction of Beclin 1 with Atg14L and UVRAG in Usp14 ${ }^{-/-} \mathrm{H} 4$ cells was significantly increased compared with that of wild-type $\mathrm{H} 4$ cells (Fig. 5H). Since Atg14L and UVRAG promote Vps34 complex activity in mediating autophagy induction and maturation, respectively, these results suggest that, in addition to regulating autophagy initiation through Atg14L complexes, K63 ubiquitination of Beclin 1 may also play a role in promoting autophagosome maturation through regulating the UVRAG-containing Vps34 complex.

\section{Akt-mediated phosphorylation regulates USP14 DUB activity toward Beclin 1}

Since Akt-mediated USP14 phosphorylation activates USP14 DUB activity (Xu et al. 2015a) and since we found that USP14 could regulate K63 ubiquitination of Beclin 1, we next examined whether USP14 might regulate K63 ubiquitination of Beclin 1 in an Akt-dependent manner. We first characterized the levels of K63 ubiquitination on Beclin 1 under starvation conditions, which lead to inactivation of Akt. We found that the K63 ubiquitination levels of endogenous Beclin 1 were increased under starvation conditions (Fig. 6A). A similar increase in K63 ubiquitination levels on exogenous transfected Beclin 1 in Beclin 1 knockdown cells was also found (Supplemental Fig. S5). Moreover, starvation-induced K63 ubiquitination of Beclin 1 was inhibited upon the expression of activated Akt (Fig. 6B).

To test whether phosphorylation of USP14 is required for its DUB activity toward K63 ubiquitination of Beclin 1 , we stably expressed wild-type USP14 or mutant USP14 in Usp14 knockout cells and examined the effect of Beclin 1 ubiquitination. As shown in Figure 6C, the expression of wild-type USP14 or the USP14-DD mutant, but not the USP14-AA mutant, was able to inhibit the K63 ubiquitination of Beclin 1. To further validate that USP14-regulated K63 ubiquitination of Beclin 1 is dependent on Akt-mediated phosphorylation, we expressed activated Akt in Usp14 knockout cells complemented with wild-type USP14 or the USP14-AA mutant. The expression of wild-type USP14 was able to inhibit ubiquitination of Beclin 1 in both the presence and absence of Myr-Akt expresssion, while that of the USP14-AA mutant failed to inhibit ubiquitination of Beclin 1 even in the presence of activated Akt (Fig. 6D), suggesting that the USP14-regulated deubiquitination of Beclin 1 is dependent on Akt-mediated phosphorylation. Moreover, the USP14-DD mutant was still able to reduce ubiquitination levels of Beclin 1 even in the presence of the Akt inhibitor MK2206, while wild-type USP14 lost its deubiquitination activity toward Beclin 1 upon inhibition of Akt (Fig. 6E). Taken together, these results suggest that Akt-mediated phosphorylation of USP14 regulates its K63 deubiquitinating activity toward Beclin 1 .
A

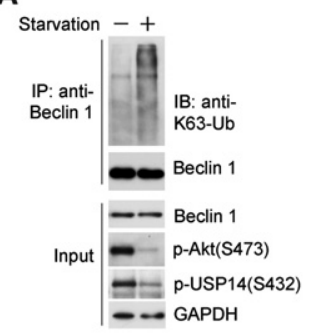

B

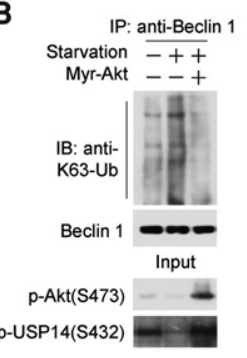

C
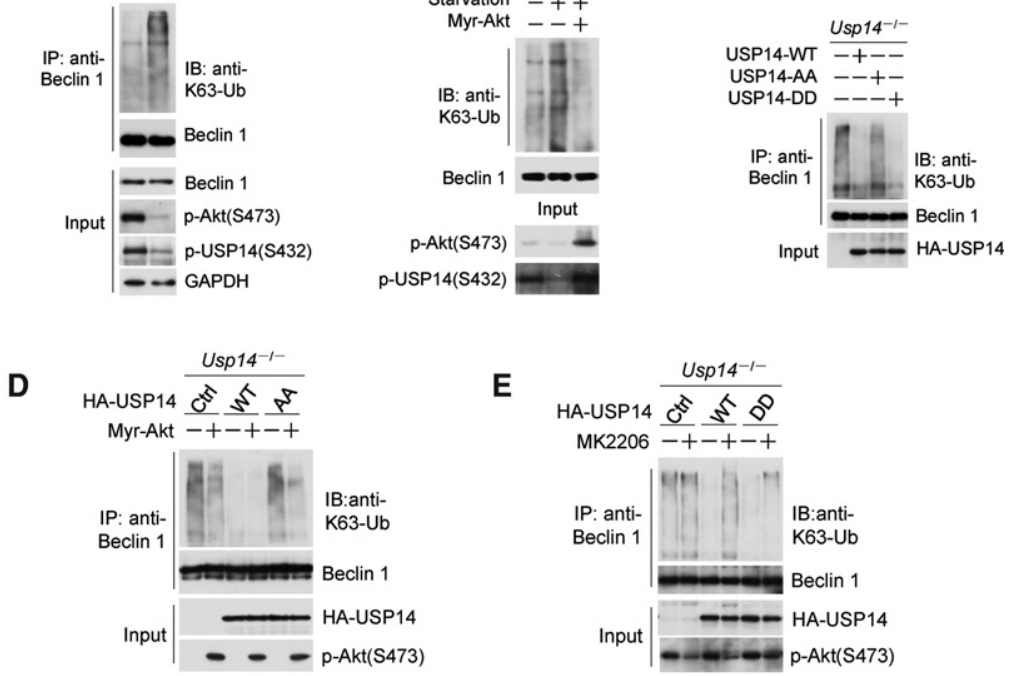

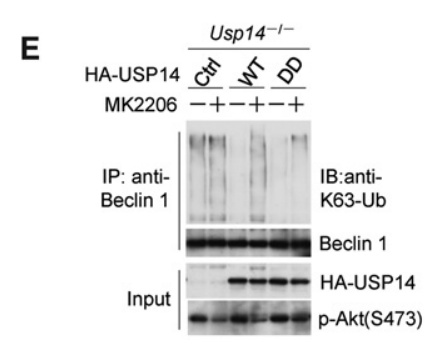

Figure 6. Akt-mediated phosphorylation regulates USP14 DUB activity toward Beclin 1. (A) H4 cells were serum-starved overnight, the lysates were analyzed by immunoprecipitation with anti-Beclin 1, and the immunocomplexes were analyzed by Western blot analysis using an anti-ubiquitin K63-specific antibody. (B) H4 cells were virally infected with a lentiviral vector for Myr-Akt for $12 \mathrm{~h}$ and then serumstarved for another $12 \mathrm{~h}$, the lysates were analyzed by immunoprecipitation with anti-Beclin 1, and the immunocomplexes were analyzed as in $A .(C)$ Usp $14^{-/-}$H4 cells were virally transfected with wild-type USP14 or the mutant as indicated for 24 $\mathrm{h}$, the lysates were analyzed by immunoprecipitation with anti-Beclin 1, and the immunocomplexes were analyzed by Western blotting. (D) Usp $14^{-/-} \mathrm{H} 4$ cells were virally transfected with wild-type USP14 or the USP14-AA mutant as indicated with or without Myr-Akt for $24 \mathrm{~h}$, the lysates were analyzed by immunoprecipitation with anti-Beclin 1, and the immunocomplexes were analyzed as in C. (E) Usp14-/- H4 cells were virally transfected with wild-type USP14 or the USP14-DD mutant as indicated for $18 \mathrm{~h}$ and then treated with or without $1 \mu \mathrm{M}$ MK2206 for another $6 \mathrm{~h}$, the lysates were analyzed by immunoprecipitation with anti-Beclin 1, and the immunocomplexes were analyzed as in $D$. 
Xu et al.

USP14 regulates autophagy by modulating K63-linked ubiquitination of Beclin 1

Since both USP14-mediated Beclin 1 deubiquitination and autophagy inhibition are dependent on Akt activation, we reasoned that Akt-activated USP14 might regulate autophagy through deubiquinating K63-linked ubiquiti- nation of Beclin 1 . To test this hypothesis, we first characterized the role of K63 ubiquitination of Beclin 1 in the regulation of autophagy by Akt. We transfected an expression vector encoding K63-only ubiquitin into H4 cells in the presence or absence of an Akt inhibitor. As shown in Figure 7A, overexpression of K63-only ubiquitin induced autophagy as indicated by the increase in the levels of
A

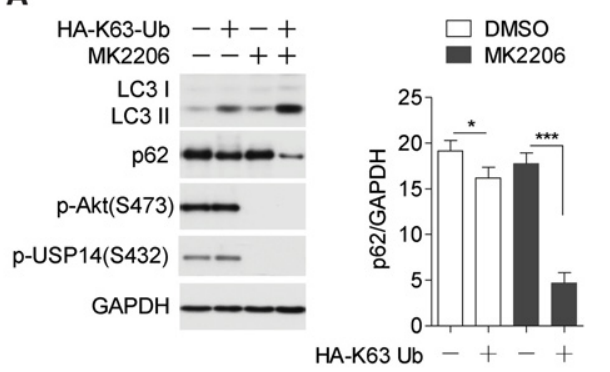

B

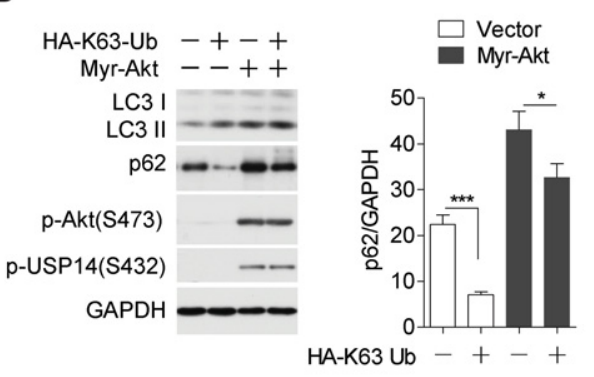

C

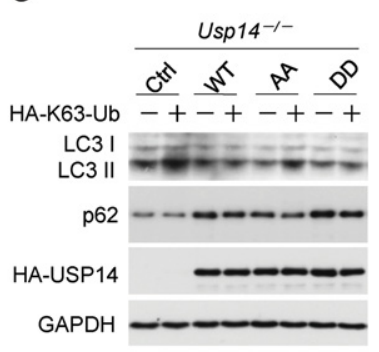

D

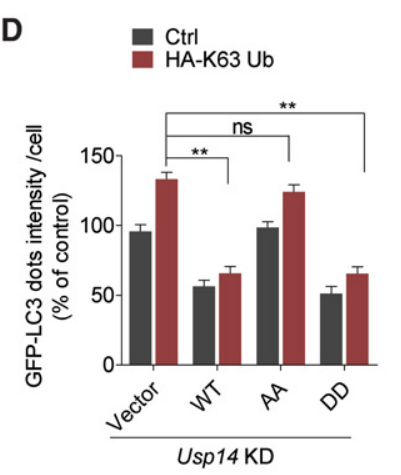

E

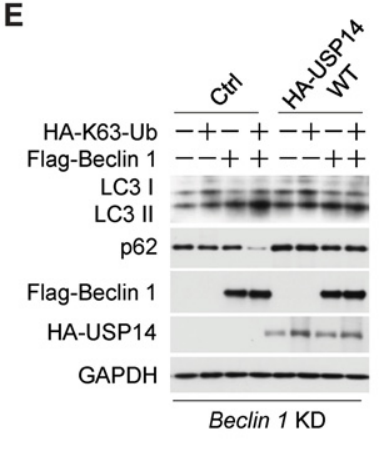

$\mathbf{F}$

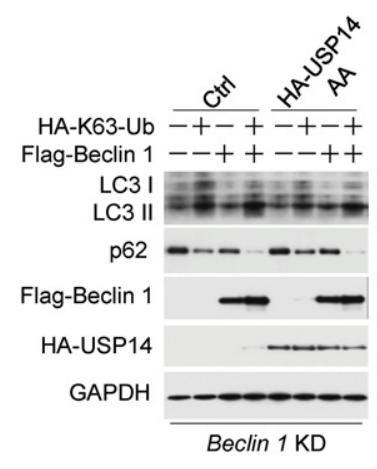

G

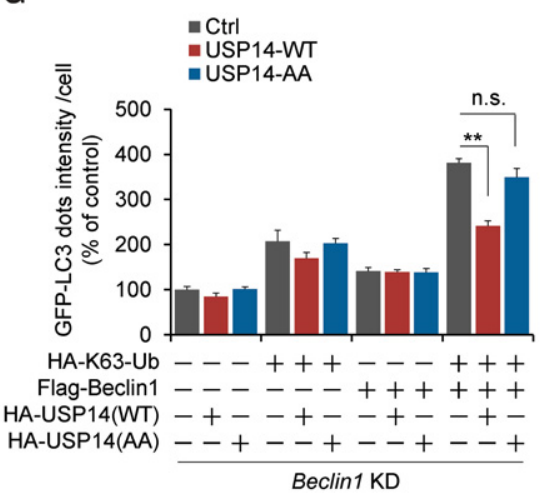

Figure 7. USP14 regulates autophagy by modulating Beclin $1 \mathrm{~K} 63$-linked ubiquitination. $(A) \mathrm{H} 4$ cells were virally infected with an expression vector for HA-tagged K63-only ubiquitin for $18 \mathrm{~h}$ and then treated with or without $1 \mu \mathrm{M}$ MK2206 for another $6 \mathrm{~h}$, and the lysates were analyzed by Western blotting. The ratios of p62/GAPDH were calculated and are shown at the right. Results are shown as means \pm SD of three independent sets of experiments. $(B) \mathrm{H} 4$ cells were virally infected with an expression vector for HA-tagged K63-only ubiquitin with or without that of Myr-Akt for $24 \mathrm{~h}$, and the lysates were analyzed by Western blotting. The ratios of p62/GAPDH were calculated and are shown at the right. Results are shown as means \pm SD of three independent sets of experiments. (C) Usp14 ${ }^{-/-} \mathrm{H} 4$ cells were virally infected with expression vectors for wild-type USP14 or the mutant as indicated with or without HA-tagged K63-only ubiquitin for $24 \mathrm{~h}$, and the lysates were analyzed by Western blotting. (D) H4-GFP-LC3 cells with Usp14 stable knockdown were virally infected with expression vectors for wild-type USP14 or the mutant as indicated with or without HA-tagged K63-only ubiquitin for $24 \mathrm{~h}$ and imaged using an ArrayScan HCS 4.0 reader. The average spot intensity in 1000 cells from each indicated sample was determined. $(E, F) \mathrm{H} 4 \mathrm{cells}$ with Beclin 1 stable knockdown were virally infected with expression vectors for Flag-tagged Beclin 1 and HA-tagged K63-only ubiquitin as indicated in the presence or absence of wild-type USP14 $(E)$ or the USP14-AA mutant $(F)$ for $24 \mathrm{~h}$, and the lysates were analyzed by Western blotting. $(G)$ H4-GFP-LC3 cells with Beclin 1 stable knockdown were virally infected as in $E$ and $F$ and then were imaged and quantified as in $D$. 
LC3-II and the decrease in the levels of p62, and this effect was further enhanced when the cells were treated with MK2206. Conversely, when coexpressed with activated Akt, the expression of K63-only ubiquitin was not able to further increase autophagy, as determined by $\mathrm{p} 62$ protein levels (Fig. 7B). These results suggest that K63 ubiquitination-promoted autophagy is negatively regulated by Akt.

To demonstrate that Akt-mediated USP14 phosphorylation inhibits autophagy through Beclin 1 K63-linked deubiquitination, we overexpressed wild-type USP14 or mutants together with K63-only ubiquitin in Usp14 knockout and knockdown $\mathrm{H} 4$ cells (Fig. 7C,D). Strikingly, both wild-type USP14 and phosphomimic mutant USP14 (USP14-DD), but not USP14-AA, were able to inhibit K63 ubiquitination-induced autophagy as determined by both Western blotting and GFP-LC3 assay (Fig. 7C,D). To further verify the role of USP14-mediated Beclin $1 \mathrm{~K} 63$ linked deubiquitination in autophagy regulation, we transfected expression vectors for K63-only ubiquitin and Beclin 1 in Beclin 1 stable knockdown $\mathrm{H} 4$ cells (Xu et al. 2015b) in the presence or absence of USP14. As shown in Figure 7E, coexpression of K63-only ubiquitin and Beclin 1 activates autophagy activity, as determined by the reduction in p62 protein levels and increase in LC3-II protein levels, while expressing K63-only ubiquitin or Beclin 1 alone only slightly increased LC3-II levels. However, the activation of autophagy activity by coexpression of K63-only ubiquitin and Beclin 1 was totally blocked when coexpressing USP14. Moreover, the AA mutant of USP14, which cannot be phosphorylated by Akt, failed to block Beclin 1 K63 ubiquitination-induced autophagy (Fig. 7F). In addition, we further verified these results using a GFP-LC3 assay on Beclin 1 stable knockdown H4-GFP-LC3 cells (Fig. 7G; Xu et al. 2015b). From these results, we conclude that Akt-mediated phosphorylation of USP14 regulates autophagy by controlling K63linked ubiquitination of Beclin 1 under normal nutritional conditions (Supplemental Fig. S6).

\section{Discussion}

Substrate selection of both UPS and autophagy involves protein ubiquitination, although the nature of the linkage and/or topology of the ubiquitination might be different. UPS targets primarily K48 ubiquitinated proteins, whereas K63 ubiquitination may provide one of the mechanisms to target proteins for degradation through autophagy $/ \mathrm{Olz}$ mann et al. 2007; Tan et al. 2008). Since ubiquitination of different linkages such as K48 or K63 is accomplished by distinct E3 ubiquitin ligases through their respective E2 enzymes under tightly regulated conditions, ubiquitination processes are unlikely to provide common mechanisms to simultaneously control both UPS and autophagy. On the other hand, DUBs can often target more than one type of ubiquitin linkage and thus are often less selective in their targeting substrates than E3 ubiquitin ligases. In this regard, since USP14 is a DUB that can trim K48 ubiquitin chains on different substrates that bind with proteasomes, it is conceivable that USP14 might be able to regulate K63 ubiquitination on different substrates. Using mass spectrometry analysis, we demonstrated the ability of USP14 to regulate K63 ubiquitination of Beclin 1, a key component of Vps34 complexes. Since USP14 can modulate K63 ubiquitination of multiple targets, our study does not rule out that other targets of USP14 can potentially also regulate autophagy. Our study demonstrates that regulation of protein deubiquitination may provide a mechanism to control the activity of both UPS and autophagy. As a key regulator that controls both UPS and autophagy, USP14 might act as a key signaling molecule in coordinating these two major intracellular proteolytic pathways.

As a critical mediator of multiple intracellular signaling pathways, Akt regulates both anabolic and catabolic mechanisms. Akt is known to negatively regulate autophagy in both mTOR-dependent (Levine and Klionsky 2004; Levine and Kroemer 2008) and mTOR-independent (Lipinski et al. 2010; Wang et al. 2012) mechanisms. Previous studies have shown that Akt can control UPS and autophagy through distinct mechanisms. Our results suggest that Akt-mediated phosphorylation of USP14 negatively regulates both UPS and autophagy. These results suggest that activated Akt in Pten-negative cancer cells might be able to stabilize a significant portion of the proteome by inhibiting both UPS and autophagy through regulating USP14 to promote tumorigenesis. Consistent with this possibility, increased expression of USP14 has been found in a variety of cancers, including multiple myeloma (Tian et al. 2014), colorectal cancer (Shinji et al. 2006), lung cancer (Wu et al. 2013), epithelial ovarian cancer (Wang et al. 2015), and endometrial cancer (Vogel et al. 2016). Future studies will be needed to elucidate the functional role of elevated USP14 expression in tumorigenesis. Finally, our results suggest the possibility of developing inhibitors of USP14 for modulating both UPS and autophagy as a strategy to promote the degradation of misfolded proteins for the treatment of neurodegenerative diseases.

\section{Materials and methods}

\section{Cell culture}

HEK293T cells were cultured in DMEM (Gibco) with 10\% (v/v) FBS (Gibco) and $1 \%$ penicillin/streptomycin. H4, H4-GFP-LC3, H4-RFP-FYVE, and H4-Usp14 ${ }^{-/-}$cells were maintained in DMEM supplemented with $10 \%$ (v/v) FBS, $1 \%$ penicillin/streptomycin, and $1 \times$ sodium pyruvate (Invitrogen).

\section{Antibodies and reagents}

The commercial antibodies used for Western blot analysis include the following: Anti-phospho-Akt (Ser473) (1:1000 dilution; no. 3787), anti-LC3B (1:1000 dilution; no. 2775), anti-USP14 (rabbit, 1:1000 dilution; no. 11931), anti-Atg14L (1:1000 dilution; no. 5504), and anti-Flag (1:1000 dilution; no. 2368) were from Cell Signaling Technology. Anti-Beclin 1 (1:1000 dilution for Western blot [sc-11427]; 1:200 dilution for immunoprecipitation [sc48341]), anti-Akt (1:1000 dilution; sc-8312), and anti-USP14 (mouse, 1:100 dilution for immunoprecipitation; sc-393872) were from Santa Cruz Biotechnology. Anti-Myc (1:1000 dilution; 
16286-1-AP), anti-HA (1:1000 dilution; 51064-2-AP) anti-Rubion (1:1000 dilution; 21444-1-AP), and anti-GAPDH (1:10,000 dilution; 60004-1-Ig) were from Proteintech. Anti-Tubulin (1:10,000 dilution; PM054), anti-UVRAG (1:1000 dilution; PD027), and anti-p62 (1:5000; PM045) were from MBL. Anti-ubiquitin Lys63-specific antibody (1:2000 dilution; no. 2210353) was from Milllipore. Mouse monoclonal anti-Flag (1:500 for immunoprecipitation; F1804), anti-Myc, and anti-HA affinity gel (E6779) were from Sigma-Aldrich. IU1 (S7134), MK2206 (S1078), E64D (S7379), rapamycin (S1039), and MG132 (S2619) were from Selleckchem.

\section{Plasmids and siRNA transfection}

cDNAs for USP14 and a constitutively active form of Akt (MyrAkt) were cloned into pcDNA3.1 using Phanta Max Super-Fidelity DNA polymerase (Vazyme Biotech Co., Ltd.) and ClonExpress II cloning kit (Vazyme Biotech Co., Ltd.). Mutagenesis was performed using the MutExpress II mutagenesis kit (Vazyme Biotech Co., Ltd.). Cells were transfected with plasmid DNA using PolyJet DNA in vitro transfection reagent (Signagen Laboratories) according to the manufacturer's instructions. The sequences of siRNAs used in this study were as follows: siUsp14-a (5'-GGA GAAAUUUGAAGGUGUA-3'), siUsp14-b (5'-GCAGCCCUUA GAGAUUUGU- $3^{\prime}$ ), and siUsp14-c (5'-GCCUCGCAGAGUUG AAAUA-3'). siRNA transient transfections were performed using HiPerFect transfection reagent (Qiagen) according to the manufacturer's instructions.

\section{Generation of knockdown and reconstitution lines}

H4 and H4-GFP-LC3 cells were stably infected with shRNA against Usp14 3' untranslated region (UTR) (CTTTAGAGGAA GACACATA), Beclin $13^{\prime}$ UTR (CTCTGTGTTAGAGATATGA) (Xu et al. 2015b), or scramble control in the pLVX lentiviral background. Lentiviral particles were made according to the manufacturer's instructions (Clontech). Viral supernatants were collected $48 \mathrm{~h}$ after transfection. Cleared supernatant was filtered through a $0.45-\mu \mathrm{m}$ filter. Polybrene $(8 \mu \mathrm{g} / \mathrm{mL})$ was supplemented with viral supernatants. Twenty-four hours after infection, cells stably expressing shRNA were obtained by selection with $10 \mu \mathrm{g} / \mathrm{mL}$ puromycin (Invivogen). Usp14 3' UTR shRNA-expressing H4 or H4GFP-LC3 cells and Usp14 ${ }^{-1-} \mathrm{H} 4$ cells (Xu et al. 2015a) were infected with lentiviral particles expressing HA-USP14 (wild-type or mutant). Polyclonal populations were screened until wildtype and mutant lines were generated that had near-endogenous USP14 reconstitution levels.

\section{Vps34 lipid kinase assay and protein-lipid blot assay}

Vps34/Beclin 1 complexes were immunoprecipitated by antibody against Beclin 1. The immunoprecipitated beads were washed three times and added with $0.1 \mathrm{mg} / \mathrm{mL}$ PI:3PS and $25 \mu \mathrm{M}$ ATP in $50 \mu \mathrm{L}$ of reaction buffer (50 mM HEPES at $\mathrm{pH} 7.5,50 \mathrm{mM}$ $\mathrm{NaCl}, 3 \mathrm{mM} \mathrm{MgCl} 2,0.025 \mathrm{mg} / \mathrm{mL} \mathrm{BSA}$ ) for $1 \mathrm{~h}$ at $25^{\circ} \mathrm{C}$. The production of PtdIns3P was detected by protein-lipid blot assay as reported (Liu et al. 2011; Xu et al. 2015b). Briefly, lipid extracted from the reaction was spotted onto a Hybond C-extra membrane (Amersham) using a Bio-DOTTM apparatus (Bio-Rad). The membrane was allowed to dry overnight in the dark. Next, the membrane was incubated with blocking buffer ( $5 \%$ BSA in TBST) for $1 \mathrm{~h}$, washed once in TBST for $30 \mathrm{~min}$, and incubated with protein buffer (1 mg of GST-tagged PX-p40 domain protein, which binds to PtdIns3P, per $1 \mathrm{~mL}$ of TBST with $5 \% \mathrm{BSA}$ ) overnight at $4^{\circ} \mathrm{C}$. The membrane was then washed in TBST three times for
30 min each, incubated with anti-GST (Sigma) in 5\% BSA buffer for $4 \mathrm{~h}$, washed in TBST for three changes for $5 \mathrm{~min}$ each, incubated with secondary antibody for $1 \mathrm{~h}$, and washed in TBST for three changes for $5 \mathrm{~min}$ each. All incubations were at room temperature unless noted otherwise. The signals were visualized with ECL.

\section{Mass spectrometry and data analysis}

The quantitative analysis of ubiquitinated proteome change in the absence or presence of USP14 in H4 cells expressing FlagHis-K63-only ubiquitin was performed by TMT isobaric labeling followed by shotgun analysis. The immunoprecipitation for Flag followed by His pull-down was performed to obtain ubiquitinated proteome. The proteins pulled down from control cells, or cells overexpressing wild-type USP14 or the USP14-AA mutant were trypsin-digested and labeled with 126-TMT, 127-TMT, and 129TMT labeling reagent (Thermo Scientific), respectively, as the instructions described. The same amount of peptides was mixed with each TMT tag, and the resulting mixture of peptides was analyzed on a Q Exactive Hybrid Quadrupole Orbitrap mass spectrometer. Three replicates were performed. The protein identification and quantification were done by Thermo Proteome discoverer (version 1.4). The peptide false positive rate was controlled to be $<1 \%$. The peak integration tolerance was set as 10 $\mathrm{ppm}$. Only unique peptides were used for protein quantitation. The isobaric tag purity correction was performed. The labeling efficiency was measured and was $>99 \%$. The average ratios of each protein from three replicates were used for analysis.

The binding partners of endogenous Beclin 1 with overexpression of K63 ubiquitin were identified by mass spectrometry. The proteins obtained by immunoprecipitation against Beclin 1 in cells with or without K63 ubiquitin overexpression were trypsin-digested on beads. The resulting peptides were analyzed on a Thermo Scientific Orbitrap Fusion Tribrid mass spectrometer. Three replicates were performed. The protein identification and quantification were done by MaxQuant (Cox and Mann 2008). The tandem mass spectra were searched against the UniProt human protein database and a set of commonly observed contaminants. The precursor mass tolerance was set as $20 \mathrm{ppm}$, and the fragment mass tolerance was set as $0.5 \mathrm{Da}$. The cysteine carbamidomethylation was set as a static modification, and the methionine oxidation was set as a variable modification. The false discovery rate at the peptide spectrum match level and protein level was controlled to be $<1 \%$. The unique peptides plus razor peptides were included for quantification. The summed peptide intensities were used for protein quantification.

\section{In vitro deubiquitination assay}

For preparation of ubiquitinated Beclin 1 as the substrate for the in vitro deubiquitination assay, HEK293T cells were transfected with expression vectors of HA-K63-ubiquitin and Flag-Beclin 1 for $24 \mathrm{~h}$, and ubiquitinated Beclin 1 was purified from the cell extracts with anti-Flag affinity column in lysis buffer $(150 \mathrm{mM}$ $\mathrm{NaCl}, 50 \mathrm{mM}$ Tris- $\mathrm{HCl}$ at $\mathrm{pH} 7.4,1 \% \mathrm{NP}-40$, protease inhibitor cocktail, $100 \mu \mathrm{g} / \mathrm{mL}$ PMSF, $1 \mathrm{mM} \mathrm{Na} \mathrm{VO}_{4}, 50 \mathrm{mM} \mathrm{NaF}$ ). After extensive washing with the lysis buffer, the proteins were eluted with Flag peptides (Sigma). The recombinant USP14 and USP14-S432E were obtained as previously reported (Xu et al. 2015a). Ubiquitinated Beclin 1 protein was incubated with recombinant USP14 in a deubiquitination buffer $150 \mathrm{mM}$ Tris$\mathrm{HCl}$ at $\mathrm{pH} 8.0,50 \mathrm{mM} \mathrm{NaCl}, 1 \mathrm{mM}$ EDTA, $10 \mathrm{mM} \mathrm{DTT}, 5 \%$ glycerol) for $2 \mathrm{~h}$ at $37^{\circ} \mathrm{C}$. 


\section{Cell imaging and statistical analysis}

Cells were fixed with $4 \%$ paraformaldehyde (Sigma) and stained with $3 \mu \mathrm{g} / \mathrm{mL}$ DAPI (Sigma). Image data were collected with an ArrayScan HCS 4.0 reader with a 203 objective (Cellomics ArrayScan VTI) for DAPI-labeled nuclei and GFP/RFP-tagged intracellular proteins. Error bars for microscopy are presented as the standard deviation of triplicate samples. Error bars for Western blot analysis represent the standard deviation between densitometry data from three unique experiments. Student's $t$-test was used as statistical analysis by using GraphPad Prism.

\section{Acknowledgments}

This work was supported in part by grants from the Chinese Academy of Sciences, the China Ministry of Science and Technology Program (2014ZX09102001-002), the State Key Program of National Natural Science of China (no. 31530041), the Global Research Laboratory Program (GRL, NRF-2010-00341) (to J.Y.), and the Ministry of Science and Technology (MEST) in Korea and the Shanghai Natural Science Foundation 16ZR1443900 (to B.S.).

\section{References}

Bence NF, Bennett EJ, Kopito RR. 2005. Application and analysis of the GFPu family of ubiquitin-proteasome system reporters. Method Enzymol 399: 481-490.

Borodovsky A, Kessler BM, Casagrande R, Overkleeft HS, Wilkinson KD, Ploegh HL. 2001. A novel active site-directed probe specific for deubiquitylating enzymes reveals proteasome association of USP14. EMBO / 20: 5187-5196.

Burgering BM, Coffer PJ. 1995. Protein kinase B (c-Akt) in phosphatidylinositol-3-OH kinase signal transduction. Nature 376: 599-602.

Ciechanover A, Kwon YT. 2015. Degradation of misfolded proteins in neurodegenerative diseases: therapeutic targets and strategies. Exp Mol Med 47: e147.

Cox J, Mann M. 2008. MaxQuant enables high peptide identification rates, individualized p.p.b.-range mass accuracies and proteome-wide protein quantification. Nat Biotechnol 26: 1367-1372.

Franke TF, Yang SI, Chan TO, Datta K, Kazlauskas A, Morrison DK, Kaplan DR, Tsichlis PN. 1995. The protein kinase encoded by the Akt proto-oncogene is a target of the PDGF-activated phosphatidylinositol 3-kinase. Cell 81: 727-736.

Hu M, Li P, Song L, Jeffrey PD, Chenova TA, Wilkinson KD, Cohen RE, Shi Y. 2005. Structure and mechanisms of the proteasome-associated deubiquitinating enzyme USP14. EMBO I 24: 3747-3756.

Itakura E, Kishi C, Inoue K, Mizushima N. 2008. Beclin 1 forms two distinct phosphatidylinositol 3-kinase complexes with mammalian Atg14 and UVRAG. Mol Biol Cell 19: 5360-5372.

Jung H, Kim BG, Han WH, Lee JH, Cho JY, Park WS, Maurice MM, Han JK, Lee MJ, Finley D, et al. 2013. Deubiquitination of Dishevelled by Usp14 is required for Wnt signaling. Oncogenesis 2: $\mathrm{e} 64$.

Komander D, Rape M. 2012. The ubiquitin code. Annu Rev Biochem 81: 203-229.

Koulich E, Li X, DeMartino GN. 2008. Relative structural and functional roles of multiple deubiquitylating proteins associated with mammalian 26S proteasome. Mol Biol Cell 19: 1072-1082.

Lee BH, Lee MJ, Park S, Oh DC, Elsasser S, Chen PC, Gartner C, Dimova N, Hanna J, Gygi SP, et al. 2010. Enhancement of pro- teasome activity by a small-molecule inhibitor of USP14. Nature 467: 179-184.

Lee BH, Lu Y, Prado MA, Shi Y, Tian G, Sun S, Elsasser S, Gygi SP, King RW, Finley D. 2016. USP14 deubiquitinates proteasomebound substrates that are ubiquitinated at multiple sites. $\mathrm{Na}$ ture 532: 398-401.

Levine B, Klionsky DJ. 2004. Development by self-digestion: molecular mechanisms and biological functions of autophagy. Dev Cell 6: 463-477.

Levine B, Kroemer G. 2008. Autophagy in the pathogenesis of disease. Cell 132: 27-42.

Lipinski MM, Hoffman G, Ng A, Zhou W, Py BF, Hsu E, Liu X, Eisenberg J, Liu J, Blenis J, et al. 2010. A genome-wide siRNA screen reveals multiple mTORC1 independent signaling pathways regulating autophagy under normal nutritional conditions. Dev Cell 18: 1041-1052.

Liu J, Xia H, Kim M, Xu L, Li Y, Zhang L, Cai Y, Norberg HV, Zhang T, Furuya T, et al. 2011. Beclin1 controls the levels of p53 by regulating the deubiquitination activity of USP10 and USP13. Cell 147: 223-234.

Matsunaga K, Saitoh T, Tabata K, Omori H, Satoh T, Kurotori N, Maejima I, Shirahama-Noda K, Ichimura T, Isobe $\mathrm{T}$, et al. 2009. Two Beclin 1-binding proteins, Atg14L and Rubicon, reciprocally regulate autophagy at different stages. Nat Cell Biol 11: 385-396.

Mizushima N, Yoshimori T, Levine B. 2010. Methods in mammalian autophagy research. Cell 140: 313-326.

Olzmann JA, Li L, Chudaev MV, Chen J, Perez FA, Palmiter RD, Chin LS. 2007. Parkin-mediated K63-linked polyubiquitination targets misfolded DJ-1 to aggresomes via binding to HDAC6. J Cell Biol 178: 1025-1038.

Ordureau A, Sarraf SA, Duda DM, Heo JM, Jedrychowski MP, Sviderskiy VO, Olszewski JL, Koerber JT, Xie T, Beausoleil SA, et al. 2014. Quantitative proteomics reveal a feedforward mechanism for mitochondrial PARKIN translocation and ubiquitin chain synthesis. Mol Cell 56: 360-375.

Russell RC, Tian Y, Yuan H, Park HW, Chang YY, Kim J, Kim H, Neufeld TP, Dillin A, Guan KL. 2013. ULK1 induces autophagy by phosphorylating Beclin-1 and activating VPS34 lipid kinase. Nat Cell Biol 15: 741-750.

Sarraf SA, Raman M, Guarani-Pereira V, Sowa ME, Huttlin EL, Gygi SP, Harper JW. 2013. Landscape of the PARKIN-dependent ubiquitylome in response to mitochondrial depolarization. Nature 496: 372-376.

Shinji S, Naito Z, Ishiwata S, Ishiwata T, Tanaka N, Furukawa K, Suzuki H, Seya T, Matsuda A, Katsuta M, et al. 2006. Ubiquitin-specific protease 14 expression in colorectal cancer is associated with liver and lymph node metastases. Oncol Rep 15: 539-543.

Tan JM, Wong ES, Kirkpatrick DS, Pletnikova O, Ko HS, Tay SP, Ho MW, Troncoso J, Gygi SP, Lee MK, et al. 2008. Lysine 63linked ubiquitination promotes the formation and autophagic clearance of protein inclusions associated with neurodegenerative diseases. Hum Mol Genet 17: 431-439.

Tian Z, D'Arcy P, Wang X, Ray A, Tai YT, Hu Y, Carrasco RD, Richardson P, Linder S, Chauhan D, et al. 2014. A novel small molecule inhibitor of deubiquitylating enzyme USP14 and UCHL5 induces apoptosis in multiple myeloma and overcomes bortezomib resistance. Blood 123: 706-716.

Vaden JH, Bhattacharyya BJ, Chen PC, Watson JA, Marshall AG, Phillips SE, Wilson JA, King GD, Miller RJ, Wilson SM. 2015. Ubiquitin-specific protease 14 regulates c-Jun $\mathrm{N}$-terminal kinase signaling at the neuromuscular junction. Mol Neurodegener 10: 3 . 
Vogel RI, Pulver T, Heilmann W, Mooneyham A, Mullany S, Zhao X, Shahi M, Richter J, Klein M, Chen L, et al. 2016. USP14 is a predictor of recurrence in endometrial cancer and a molecular target for endometrial cancer treatment. Oncotarget 7: 30962-30976.

Wang RC, Wei Y, An Z, Zou Z, Xiao G, Bhagat G, White M, Reichelt J, Levine B. 2012. Akt-mediated regulation of autophagy and tumorigenesis through Beclin 1 phosphorylation. Science 338: 956-959.

Wang Y, Wang J, Zhong J, Deng Y, Xi Q, He S, Yang S, Jiang L, Huang M, Tang C, et al. 2015. Ubiquitin-specific protease 14 (USP14) regulates cellular proliferation and apoptosis in epithelial ovarian cancer. Med Oncol 32: 379.

Wu N, Liu C, Bai C, Han YP, Cho WC, Li Q. 2013. Over-expression of deubiquitinating enzyme USP14 in lung adenocarcinoma promotes proliferation through the accumulation of $\beta$ catenin. Int J Mol Sci 14: 10749-10760.
Xu D, Shan B, Lee BH, Zhu K, Zhang T, Sun H, Liu M, Shi L, Liang W, Qian L, et al. 2015a. Phosphorylation and activation of ubiquitin-specific protease-14 by Akt regulates the ubiquitin-proteasome system. Elife 4: e10510.

Xu D, Zhang T, Xiao J, Zhu K, Wei R, Wu Z, Meng H, Li Y, Yuan J. 2015b. Modification of BECN1 by ISG15 plays a crucial role in autophagy regulation by type I IFN/interferon. Autophagy 11: 617-628.

Zhang L, Yu J, Pan H, Hu P, Hao Y, Cai W, Zhu H, Yu AD, Xie X, Ma D, et al. 2007. Small molecule regulators of autophagy identified by an image-based high-throughput screen. Proc Natl Acad Sci 104: 19023-19028.

Zhong Y, Wang QJ, Li X, Yan Y, Backer JM, Chait BT, Heintz N, Yue Z. 2009. Distinct regulation of autophagic activity by Atg14L and Rubicon associated with Beclin 1-phosphatidylinositol-3-kinase complex. Nat Cell Biol 11: 468-476. 


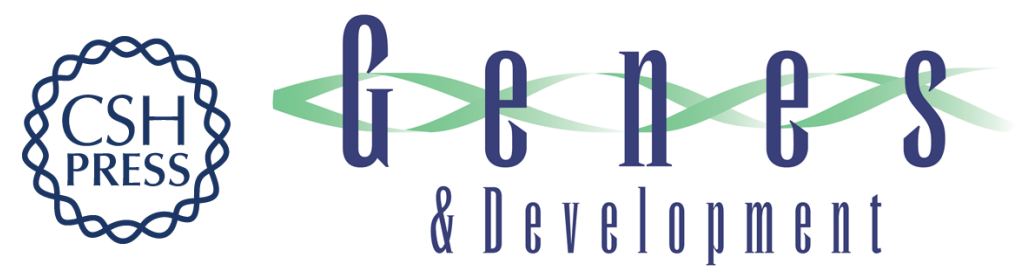

\section{USP14 regulates autophagy by suppressing K63 ubiquitination of Beclin 1}

Daichao Xu, Bing Shan, Huawang Sun, et al.

Genes Dev. 2016, 30:

Access the most recent version at doi:10.1101/gad.285122.116

\section{Supplemental http://genesdev.cshlp.org/content/suppl/2016/08/19/30.15.1718.DC1 Material}

References This article cites 35 articles, 8 of which can be accessed free at: http://genesdev.cshlp.org/content/30/15/1718.full.html\#ref-list-1

Creative This article is distributed exclusively by Cold Spring Harbor Laboratory Press for the first Commons six months after the full-issue publication date (see

License http://genesdev.cshlp.org/site/misc/terms.xhtml). After six months, it is available under a Creative Commons License (Attribution-NonCommercial 4.0 International), as described at http://creativecommons.org/licenses/by-nc/4.0/.

Email Alerting Receive free email alerts when new articles cite this article - sign up in the box at the top Service right corner of the article or click here.

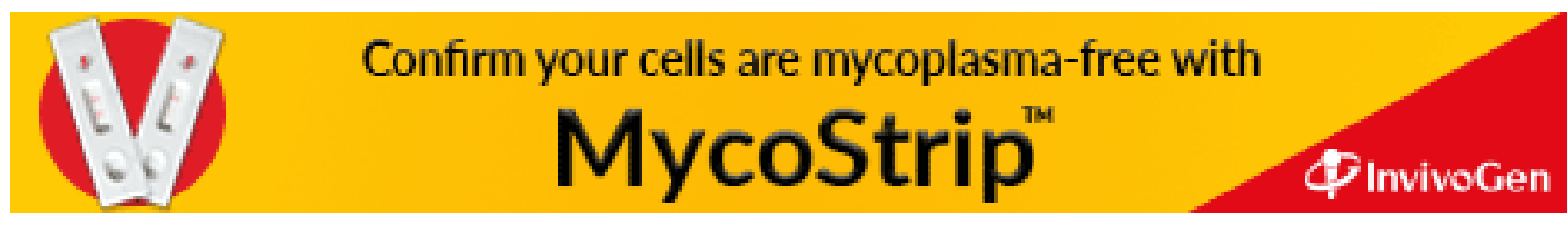

